\title{
Early response in cognitive-behavior therapy for syndromes of medically unexplained symptoms
}

\author{
Maria Kleinstäuber ${ }^{1,2^{*}}$ (D), Michael J. Lambert ${ }^{3}$ and Wolfgang Hiller ${ }^{4}$
}

\begin{abstract}
Background: Early dramatic treatment response suggests a subset of patients who respond to treatment before most of it has been offered. These early responders tend to be over represented among those who are well at termination and at follow-up. Early response patterns in psychotherapy have been investigated only for a few of mental disorders so far. The main aim of the current study was to examine early response after five therapypreparing sessions of a cognitive behavior therapy (CBT) for syndromes of medically unexplained symptoms (MUS).
\end{abstract}

Methods: In the context of a randomized, waiting-list controlled trial 48 patients who suffered from $\geq 3$ MUS over $\geq 6$ months received 5 therapy-preparing sessions and 20 sessions of CBT for somatoform disorders. They completed self-report scales of somatic symptom severity (SOMS-7 T), depression (BDI-II), anxiety (BSI), illness anxiety and behavior (IAS) at pre-treatment, after 5 therapy-preparing sessions (FU-5P) and at therapy termination (FU-20 T).

Results: The current analyses are based on data from the treatment arm only. Repeated measure ANOVAs revealed a significant decrease of depression $(d=0.34)$, anxiety $(d=0.60)$, illness anxiety $(d=0.38)$ and illness behavior $(d=0.42)$, but no change of somatic symptom severity $(d=-0.03)$ between pre-treatment and FU-5P. Hierarchical linear multiple regression analyses showed that symptom improvements between pre-treatment and FU-5P predict a better outcome at therapy termination for depression and illness anxiety, after controlling for pre-treatment scores. Mixed-effect ANOVAs revealed significant group*time interaction effects indicating differences in the course of symptom improvement over the therapy between patients who fulfilled a reliable change (i.e., early response) during the 5 therapy-preparing sessions and patients who did not reach an early reliable change. Demographic or clinical variables at pre-treatment were not significantly correlated with differential scores between pre-treatment and FU-5P $(-.23 \leq r \leq .23)$.

Conclusions: Due to several limitations (e.g., small sample size, lack of a control group) the results of this study have to be interpreted cautiously. Our findings show that reliable changes in regard to affective-cognitive and behavioral variables can take place very early in CBT of patients with distressing MUS. These early changes seem to be predictive of the outcome at therapy termination. Future studies are needed in order to replicate our results, and to identify mechanisms of these early response patterns in somatoform patients.

Trial registration: ISRCTN. ISRCTN17188363. Registered retrospectively on 29 March 2007.

Keywords: Early response, Cognitive-behavior therapy, Medically unexplained symptoms, CBT, MUS

\footnotetext{
*Correspondence: maria.kleinstaeuber@staff.uni-marburg.de;

m.kleinstauber@auckland.ac.nz

${ }^{1}$ Division of Clinical Psychology and Psychotherapy, Philipps-University,

Gutenbergstr. 18, D-35037 Marburg, Germany

${ }^{2}$ ssychological Medicine, School of Medicine, Faculty of Medical and Health

Sciences, University of Auckland, Hospital Bldg. 599, 2 Park Rd, Grafton

Auckland, Auckland 1023, New Zealand

Full list of author information is available at the end of the article
} 


\section{Background}

The term "early response" originates from pharmacological research, in particular research of inexplicable relapse during continuation treatment [1]. The Columbia Group led by Frederic M. Quitkin [2] defined the phenomenon of the "true-drug initial response pattern" which means that a delayed persistent response predicts a positive long-term outcome of an antidepressant treatment. In the contrary, an early response going along with patterns of fluctuating improvement is rather associated with relapse [3] and rather attributable to placebo effects [2].

In psychotherapy research only a few studies [4-8] have investigated the timing of response in psychotherapy. In contrast to pharmacotherapy, they demonstrated that early response patterns are related to better intermediate and long-term outcomes. One of the earliest studies on rapid response in psychotherapy was conducted by Fennell and Teasdale [4]. The authors compared a sample of patients with major depressive disorder (MDD) who responded to cognitive-behavior therapy (CBT) with a median change in depressive symptoms (46\%) or more with MDD patients whose response was lower than a median improvement before the fourth therapy session. The results showed that the rapid responders maintained their early therapy gains throughout the active treatment period. Moreover, those who demonstrated an early therapy response continued to do better until the end of the treatment than those who initially hardly improved. Patients who were below the median change of depressive symptoms after the first three sessions had outcomes at the end of therapy which were similar to those of control participants who received treatment as usual (TAU). Finally authors could demonstrate that the extent of early change in depressive symptoms strongly predicted post-treatment outcome in the CBT but not in the TAU control group.

In another study Renaud et al. [5] demonstrated (in a group of depressive adolescents) that early responders compared to individuals whose score did not change or deteriorated after the first two treatment weeks had better outcomes at the end of therapy and at a 1- and 2-year follow-up. This finding was similar across three different kinds of therapy (CBT, systemic behavioral family therapy, and nondirective supportive treatment). Similarly Haas et al. [6] showed in an outpatient psychotherapy sample of 147 patients with mixed diagnoses of mental disorders that a rapid response across sessions 1 through 3 predicted better long-term outcomes, even after analyses controlled for initial symptom severity. Furthermore there are two studies $[7,8]$ in which patterns of response in early therapy sessions were examined as a function of patients' pre-treatment symptom severity.
Lutz et al. [7] examined a completer sample of $N=162$ depressive patients treated with different treatment protocols (cognitive behavior therapy, interpersonal therapy, imipramine plus clinical management, pill-placebo plus clinical management) were analyzed with growth mixture models in order to identify meaningful patterns of early change of depression severity. Type of treatment had low explanatory power for the change of depressive symptoms at termination and follow-up. In contrast, patterns of early change as well as pre-treatment overall symptom severity were significant predictors of short- and long-term outcomes. In particular results showed that patients with moderate to severe depression and rapid early improvement had the highest rate of reliable improvement $(100 \%)$ at both 16 week and 18-month follow-up. In contrast, the lowest rate of improvement at termination (34\%) was found in patients who had a mild to moderate level of depression at pre-treatment and who demonstrated a moderate improvement during early therapy sessions. However, these patients showed further improvement during the followup phase. In another study by Lutz et al. [8] a large sample of psychotherapy outpatients with mixed diagnoses was analyzed with growth mixture models. According to their level of pre-treatment depression patients were assigned to one of three subgroups. Group 1 indicated mild, group 2 moderate, and group 3 severe depressive symptoms at pre-treatment. For each group the authors identified 4 different response patterns. Whereas some of these response patterns were less similar between the three groups, there was one pattern which appeared in all three subgroups and which was characterized by only slight changes during the first five sessions. Interestingly this response pattern was associated with a low benefit from therapy at post-treatment assessment in all three groups.

In two of the studies mentioned above $[4,8]$ the authors tried to identify predictors of early response in psychotherapy. Fennel et al. [4] could not find differences between depressive patients responding rapidly to CBT vs. patients who only slightly changed during the first two weeks of treatment in regard to demographics, clinical variables (e.g., duration, severity of symptoms), and antidepressant medication at pre-treatment. Lutz et al. [8] could identify depressive and interpersonal problems, agoraphobic symptoms and somatization, and problems at school or in the job as predictors of the identified patterns of changes in the early phase of therapy.

This summary of studies demonstrates that there is a substantial number of patients who experience an early positive change in therapy before it is completed or therapy techniques are initiated. However this phenomenon of early response has been examined only in a very 
limited number of groups of patients - mainly in depressive patients $[4,5,7]$ or patients with mixed diagnoses $[6,8]$. Other clinical samples, in which early response could be of special interest, have not been investigated in regard to this phenomenon. A clinical sample could be patients suffering from syndromes of medically unexplained physical symptoms (MUS).

Medically unexplained symptoms (MUS) are somatic symptoms that cannot or have not been sufficiently explained by organic causes after a thorough physical examination [9]. According to diagnostic criteria of the fourth revision of the Diagnostic and Statistical Manual for Mental Disorders (DSM-IV) [10], the chronic and severely distressing manifestation of MUS is a core characteristic of any one of the following somatoform disorders: somatization disorder, undifferentiated somatoform disorder, and pain disorder. According to the current revision of DSM - DSM-5 [11] - these three diagnostic categories are covered by somatic symptom disorder. Syndromes of MUS are highly prevalent in the general population [12], in primary care [13], and in medical specialist settings [14]. In longitudinal studies a high risk of symptom persistence has been demonstrated [15]. MUS and somatoform disorders are associated with high rates of comorbid mental disorders [16], high levels of symptom-related burden and disability in everyday life functioning [17]. As a consequence of extensive utilization of health care services [18] health care costs are substantially increased in this group of patients. Psychological interventions, especially cognitive behavior therapy, have been demonstrated to be effective in reducing symptom severity but also secondary outcomes (e.g., level of functional disability, depressive and anxiety symptoms) $[19,20]$.

There are several reasons why early response patterns in somatoform patients could be of special interest. Due to the somatic nature of their complaints patients with somatoform disorders partly attribute their symptoms strongly to biomedical causes [21]. Consequently for a subset of these patients it is difficult to consider psychotherapy as a helpful and appropriate treatment for their complaints. Due to this issue it is not surprising that the physician-patient relationship can be conflict-laden and characterized by problems in communication [22]. A typical feature of such communication problems is that patients appear as demanding or premeditated to pursue certain ideas (especially finding a somatic cause of their symptoms) [23]. In turn physicians can feel pressured or controlled [24]. When patients strongly complain about their symptoms physicians are likely to prescribed somatic interventions [25]. Thus it is not surprising that patients suffering from long-standing MUS often present with a long history of somatic diagnostic procedures and treatments often accompanied by feelings of disappointment and frustration, negative interpersonal relationship experiences, and hopelessness [26]. These negative experiences usually contrast with interpersonal experiences in the early process of psychotherapy and may reduce the rate of early response. To our knowledge there is only one previous study examining the phenomenon of early response in patients with MUS. Lackner, Gudleski, Krasner, Powell, and Katz [27] demonstrated that $30 \%$ of patients suffering from a specific functional somatic syndrome characterized by unexplained gastrointestinal symptoms irritable bowel syndrome (IBS) - fulfilled criteria of rapid response (affirmative response to 2 binary [Yes/No] adequate relief measures of pain and other IBS symptoms; decrease in Irritable Bowel Syndrome Severity Scale [28] total score of $\geq 50$ points after four weeks of treatment; patients who did not meet both of these criteria at week 12 were classified as non rapid responders). Rapid responders had higher symptom severity at baseline but also a better outcome in regard to symptom reduction at posttreatment in comparison to non rapid responders.

Ilardi and Craighead $[29,30]$ reviewed studies of CBT for depression in regard to common factors and demonstrated that the majority of symptomatic improvement in CBT occurs prior to the formal induction of techniques being specific to CBT. Accordingly the authors assumed that non-specific factors rather than specific techniques explain the efficacy of CBT, in particular by ameliorating patients' feelings of hopelessness at the beginning of treatment. This explanation underlines the idea that early response patterns in psychotherapy could play a special role in samples of patients with chronic somatoform disorders: New experiences of therapeutic alliance contrast with patients' previous problematic experiences of interpersonal patient-clinician relationship. This idea can be especially well investigated in the context of the German health-care system which offers patients with mental disorders five therapy-preparing sessions before therapy actually starts. During these sessions actions such as diagnostics, or creating a therapy plan but no specific therapeutic techniques are initiated.

Therefore the first aim of the current study was to examine if there are changes in general regarding the severity of somatic symptoms and symptom-related outcomes after 5 therapy-preparing sessions. The second aim was to analyze if response scores after these therapy-preparing sessions predict therapy outcomes at the end of therapy, controlling for pre-treatment level of disturbance. The third aim was to compare patients reaching a reliable change of symptoms during the early five sessions of therapy with patients who do not reach this reliable change, in regard to their symptom scores at pre-treatment, after the therapy-preparing sessions, and at the end of treatment. Finally the fourth aim was 
to examine if early changes in psychotherapy for somatoform disorders are associated with demographic and clinical variables.

\section{Methods \\ Participants}

A total of 106 participants were treated between April 2007 and September 2013 at a psychotherapeutic university outpatient clinic in Germany where a new treatment program for patients with syndromes of MUS was implemented. Patients were recruited via a waiting list for a treatment at the University Outpatient Clinic for Psychotherapy of Johannes Gutenberg-University Mainz (Germany), public media, and flyers which were placed in pharmacies, private practices of general practitioners or different kinds of medical specialists (e.g., orthopedists, gastroenterologists).

Participants being eligible for this study had to fulfill the following inclusion criteria: a) $\geq 3$ distressing MUS over the last six months or longer, b) exclusion of medical causes of the symptoms by a physician, c) comorbidity with other mental disorders was allowed, as long as MUS were considered to be the major problem, d) age between 18 and 65 years, and e) sufficient German language skills. Exclusion criteria were a) one or more of the following psychiatric conditions: current severe episode of a major depressive disorder or bipolar disorder, suicidality, eating disorder, substance use disorder, acute psychosis, brain injury, cluster A or B personality disorder, dementia or neurodegenerative disorders, or PTSD, b) use of benzodiazepines or neuroleptics, and c) psychological inpatient treatment during the last 5 years or psychological outpatient treatment addressing MUS during the last 2 years, and d) or submitted retirement request due to MUS.

\section{Study design and procedure}

This study was designed as a randomized controlled trial. Participants were admitted to the study via a twostep procedure (telephone screening, face-to-face diagnostic interview). Eligibility criteria were first checked during the telephone screening. Participants who fulfilled the criteria were asked to complete questionnaires, were invited to a diagnostic interview conducted by trained clinical psychologists based on International Diagnostic Checklists for DSM-IV [31], and were asked to sign informed consent. Excluded participants were informed about other treatment options and were offered registry for regular psychotherapy at the outpatient clinic. Participants who fulfilled inclusion criteria were randomly assigned to either the cognitive behavioral treatment group (CBT) or the waiting list control group (WCL). Patients were assigned to a therapist during the 7 days after randomization had taken place. The treatment started with 5 therapy-preparing sessions followed by 20 sessions of cognitive behavior therapy for somatoform disorders. Participants of the waiting-list control condition (WLC) started the treatment after a waiting period of 10 weeks. Randomization was stratified for sex and symptom-related disability based on the score of the Pain Disability Index (PDI; [32]) at baseline (PDI $\leq 6 ;>6)$. The randomization was conducted by a computer scientist being independent of the trial. Only patients were blinded to the randomization results. Study coordinators and therapists were informed about the randomization results immediately after randomization had been conducted. Diagnostic study personnel were also not blinded regarding random assignments.

Since previous randomized controlled trials (RCTs) comparing CBTs with waiting control groups revealed on average medium between-group effect sizes $(\mathrm{f}=0.25$, Power $[1-\beta$ error probability $]=0.80, \alpha=0.05)[19,20]$ we conducted a priori a power analysis with $G *$ Power 3 [33] which resulted in a total sample size of $N=90$. Under consideration of an average dropout rate of $15 \%$ at post-treatment which was found in previous RCTs being comparable to our trial $[19,20]$, we estimated a total sample size of $N=106$.

All data were collected via paper-pencil versions of selfrating scales before the face-to-face diagnostic interview (pre-treatment) and follow-up assessments took place after 5 diagnostic, therapy-preparing sessions (FU-5P), at the end of treatment after 20 sessions CBT (FU-20 T), and 1 year after the end of treatment. Unfortunately, there was a substantial problem with implementing the study: An additional assessment at the end of the waiting period in the WCL was missed. Consequently the study design did not allow comparing outcomes of CBT and WCL. For the current analysis we will refer only to the participants of the CBT group. Moreover due to low response rates at the 1-year follow-up we evaluated only data of the pretreatment assessment, of FU-5P, and FU-20 T. The study was approved by the Ethics Committee of the German Psychological Association (DGPs).

\section{Cognitive behavior therapy for chronic MUS.}

Before CBT started, patients received 5 individual sessions à 50 min which did not encompass therapeutic interventions, but had the following contents: exploration of somatic symptoms and comorbid mental disorders, previous somatic and other treatments, resources and coping strategies, patients' social background and biography; personality disorder diagnostics with the Structured Clinical Interview for DSM-IV Axis II personality disorders (SCID-II) [34]; behavior analyses; and the generation of a therapy plan. After these 5 preparing sessions the therapy actually started, encompassing 20 individual sessions à $50 \mathrm{~min}$ (assigned to 6 modules: 
therapeutic targets, stress management training, attention refocusing training, cognitive restructuring, changing illness behaviors, explanatory model, and relapse prevention) whose primary aim was not to reduce the severity of the somatic symptom but to improve patients' quality of life and symptom coping. Table 1 summarizes the treatment protocol which was published elsewhere [35]. All sessions were structured in a similar way: After reflecting on patients' experiences with therapeutic exercises patients conducted between sessions, the therapeutic topic of the session followed, and finally further therapeutic exercises were planned.

CBT was conducted by master's-level clinical psychologists who were either certified $\mathrm{CB}$ therapists or in the second year of their postgraduate CBT program. They received specialized training in $\mathrm{CBT}$ for somatoform patients and continuous supervision every fourth (therapypreparing as well as therapy) session. Therapy sessions were recorded and records were discussed during supervision in order to increase therapists' adherence and competence.

\section{Measures \\ Primary outcome}

In order to assess somatic symptom severity as the primary outcome, the Screening for Somatoform

Table 1 Contents of 20 sessions of the CBT for patients with somatoform disorders

\begin{tabular}{|c|c|}
\hline Module/Session & CBT for chronic MUS \\
\hline $1 / 1$ & $\begin{array}{l}\text { Therapeutic targets } \\
\text { Exploration patient's illness beliefs; developing } \\
\text { therapeutic targets }\end{array}$ \\
\hline $2 / 2-3,16-19$ & $\begin{array}{l}\text { Stress management training } \\
\text { Psychoeducation: distress and physical symptoms; } \\
\text { stress management and relaxation techniques }\end{array}$ \\
\hline $3 / 4-6$ & $\begin{array}{l}\text { Refocusing attention training } \\
\text { Psychoeducation: relationship between attention } \\
\text { and physical symptoms; focusing exercises to shift } \\
\text { attention away from physical symptoms }\end{array}$ \\
\hline $4 / 7-11$ & $\begin{array}{l}\text { Cognitive restructuring of symptom-related } \\
\text { dysfunctional cognitions } \\
\text { Psychoeducation: dysfunctional thoughts and } \\
\text { physical symptoms; identifying individuals' } \\
\text { dysfunctional symptom-related thoughts; } \\
\text { strategies of reappraisal and cognitive } \\
\text { restructuring; behavioral experiments (symptom } \\
\text { induction) for questioning dysfunctional } \\
\text { symptom-related thoughts }\end{array}$ \\
\hline $5 / 12-15$ & $\begin{array}{l}\text { Changing illness behaviors } \\
\text { Psychoeducation: illness behaviors and physical } \\
\text { symptom; symptom inductions tasks/exposure } \\
\text { exercises and cognitive strategies to reduce } \\
\text { avoidance behaviors and doctor-shopping }\end{array}$ \\
\hline $6 / 20$ & $\begin{array}{l}\text { Explanatory model and relapse prevention } \\
\text { Summary of therapy contents in an individual } \\
\text { explanatory model of MUS; relapse prevention }\end{array}$ \\
\hline
\end{tabular}

CBT cognitive behavior therapy, MUS medically unexplained symptoms
Disorders-7 T (SOMS-7 T) [36], a self-rating questionnaire in the form of a list of the 53 physical symptoms of DSM-IV and the 10th revision of the International Classification of Diseases (ICD-10) [37], was administered. The severity over the last seven days of only those symptoms that cannot or not sufficiently be explained medically was rated on a 5-point Likert scale (answering options range between 0 to 4 ). In the current study the severity index as a mean value of all symptom ratings was used. Internal consistency was demonstrated to be high for the scale (Cronbach's $\alpha=.92$ ) [36], and acceptable in the current sample $(\alpha=0.77)$.

\section{Secondary outcomes}

Depressive symptoms were assessed with the Beck Depression Inventory-II (BDI-II; 21 items, 4-point answering scale: 0 to 3) [38] which has demonstrated good internal consistency in a previous validation study $(\alpha=.86)$ [38], and also in our sample $(\alpha=.83)$. We used the anxiety subscale (6 items) of the 53-items Brief Symptom Inventory (BSI; answering scale: 5-point answering scale: 0 to 4) [39] in order to assess anxiety symptoms. Internal consistency of this subscale was shown to be good $(\alpha=.86)$ [40], but less so in our sample $(\alpha=.70)$. Finally the Illness Attitude Scales (IAS; 29 items, 5-point scale: 0 to 4) [41] were administered in order to assess illness anxiety and illness behavior. Whereas the illness anxiety subscale demonstrated excellent reliability in a previous validation ( $\alpha=.85-.87$ ) [42] but also in the current sample ( $\alpha=.93)$, the illness behavior subscale had low internal consistency in a previous study $(\alpha=.70-.80)$ [42], and in our sample $(\alpha=.66)$. It seems that the illness behavior subscale usually reveals lower internal consistency values than the illness anxiety subscale which has been shown also in other validation studies (e.g., [43]). This may be explained by the small number of items (6 items in contrast to 17 items of the illness anxiety subscale).

\section{Definition of early response}

Previous studies applied different ways of operationalizing early treatment response. Fennell and Teasdale [4] for example utilized a median split to determine response rate. Renaud et al. [5] defined early response as symptom improvement of at least $50 \%$ over a prespecified number of sessions. A very advanced method was used by Haas et al. [6] who operationalized early response as an average difference between an "expected" recovery rate (which was derived from actuarial data available from a large sample of patients) and the "actual" recovery rate of a patient. Unfortunately such slopes of expected scores are not available for the measures we used. We therefore used the reliable change index (RCI) [44]. Patients who reached the RCI after the five therapy-preparing sessions were defined as early 
responders. The RCI was calculated by the formula $\mathrm{RC}=\mathrm{x}_{2}-\mathrm{x}_{1} / \mathrm{s}_{\text {diff, }}$, where $\mathrm{x}_{1}$ represents a subject's pretest score and $\mathrm{x}_{2}$ its posttest score. $\mathrm{S}_{\text {diff }}$ is the standard error of the difference between the two test scores, calculated from the standard error of measurement $\mathrm{s}_{\mathrm{E}}$ by the formula $s_{\text {diff }}=\sqrt{ } 2 \times\left(s_{E}\right)^{2}$. For calculating $S_{E}$, the internal consistency (see Cronbach's $\alpha$ for administered scales under Measures) and the SD of the sample at baseline (see Table 3) were used.

\section{Data analysis}

The statistical analyses were conducted using IBM SPSS Statistics 24. Our analyses are based on intention to treat-analysis for which the multiple imputation procedure offered by IBM SPSS Statistics 20 was used to replace single missing values. The procedure produced five data sets using the monotone multiple imputation algorithm [45]. These five imputed data sets were then analyzed by using standard procedures used for complete data, and then by combining the results across these analyses. $P$-values below an alpha error level $=.05$ indicated significance.

First we examined if patients who completed questionnaires differed from patients who did not complete questionnaires after the 5 therapy-preparing sessions (FU-5P) and at post-treatment (FU-20 T) in regard to demographic variables (gender, age, education), clinical variables (duration of MUPS, comorbid mental disorder), and self-rating scale pre-treatment scores with $\mathrm{Chi}^{2}$-test and $t$-tests.

In order to answer our first research question we conducted a repeated measure (rm) analysis of variance (ANOVA) with the innersubject factor "time" (pre-treatment vs. FU-5P). Dependent variables were the SOMS-7 $\mathrm{T}$ severity index, mean scores of the BDI-II, BSI anxiety subscale, and IAS illness anxiety and illness behavior subscale. To determine the magnitude of within-group changes, effect sizes using Cohen's $d$ based on the pooled standard deviations (SD) and the corresponding 95\% confidence intervals (CI) were reported [46]. According to Cohen's convention effects of $\geq 0.10 /<0.50$ are small, of $\geq 0.50 /$ $<0.80$ are medium, and $\geq 0.80$ are large.

In regard to our second research question a hierarchal linear multiple regression analysis was conducted separately for each outcome for which a statistically significant change between pre-treatment and the 5th therapypreparing session had been found (BDI, BSI-anxiety, IAS-illness anxiety, IAS-illness behavior). The predicted variable in each regression analysis was the posttreatment score (FU-20 T) of the outcome respectively. As predicting variables we entered the pre-treatment score of the outcome in the first step of the regression analysis, and additionally the difference score between the pre-treatment and FU-5P score in the analysis' second step.

Our third research question required dividing the sample in two groups, group 1 fulfilled the $\mathrm{RCI}(\mathrm{RCI}+)$ and group 2 did not fulfill the RCI (RCI-). This group splitting was conducted in regard to each outcome for which a statistically significant change between pre-treatment and the 5th therapy-preparing session had been found (BDI, BSI-anxiety, IAS-illness anxiety, IAS-illness behavior). We then calculated mixed effect 2 (RCI+ vs. RCI-) $\times 3$ (baseline vs. FU-5P vs. FU-20 T) ANOVAs. In order to break up significant main and interaction effects we calculated Bonferroni-corrected post-hoc comparisons of cell means.

Finally, in order to examine the fourth research question we conducted bivariate Pearson product-moment or Spearman rank (for sex, educational level, comorbidity) correlation analyses between demographic/clinical variables, pre-treatment scores of self-rating scales and the residual gain score (RGS) based on the difference between pre-treatment and FU-5P. These correlation analyses were conducted only for RGSs based on variables for which a significant change between pre-treatment and FU-5P had been demonstrated (BDI, BSI-anxiety, IAS-illness anxiety, IAS-illness behavior). RGSs were calculated to handle measurement error of repeated administration of the instruments and the initial difference between individuals at pretreatment [47], since preanalyses showed a high correlation between the pretreatment score and difference values between baseline and FU-P5 for each outcome (BDI: $r=.57, p<.001$; BSI-anxiety: $r=.79, p<.001$; IAS-illness anxiety: $r=.41$, $p=.004$; IAS-illness behavior: $r=.65, p<.001)$. RGSs were calculated by the formula RGS $=z_{2}-\left(z_{1} * r_{1,2}\right)$, where $z_{2}$ is the standardized post-treatment score, $z_{1}$ the standardized pre-treatment score, and $r_{1,2}$ the Pearson product-moment correlation between raw scores at preand post-treatment [47]. RGSs were reversed such that positive scores indicate improvement and negative scores deterioration.

\section{Results}

\section{Participant characteristics}

Since only participants from the treatment group were included to the analyses this section will not address participants from the control group. The flowchart in Fig. 1 shows that 48 patients were randomly assigned to the treatment group. The sample was aged on average 40.9 years $(S D=14.12$, range: $20-65$ years $)$ and $64.6 \%$ $(n=31)$ were females. Moreover $41.7 \%$ had a secondary educational level or lower according to the International Standard Classification of Education (ISCED 97) [48]. In the sample $39(81.3 \%)$ patients fulfilled the criteria of an undifferentiated somatoform disorder, 4 patients $(8.3 \%)$ 


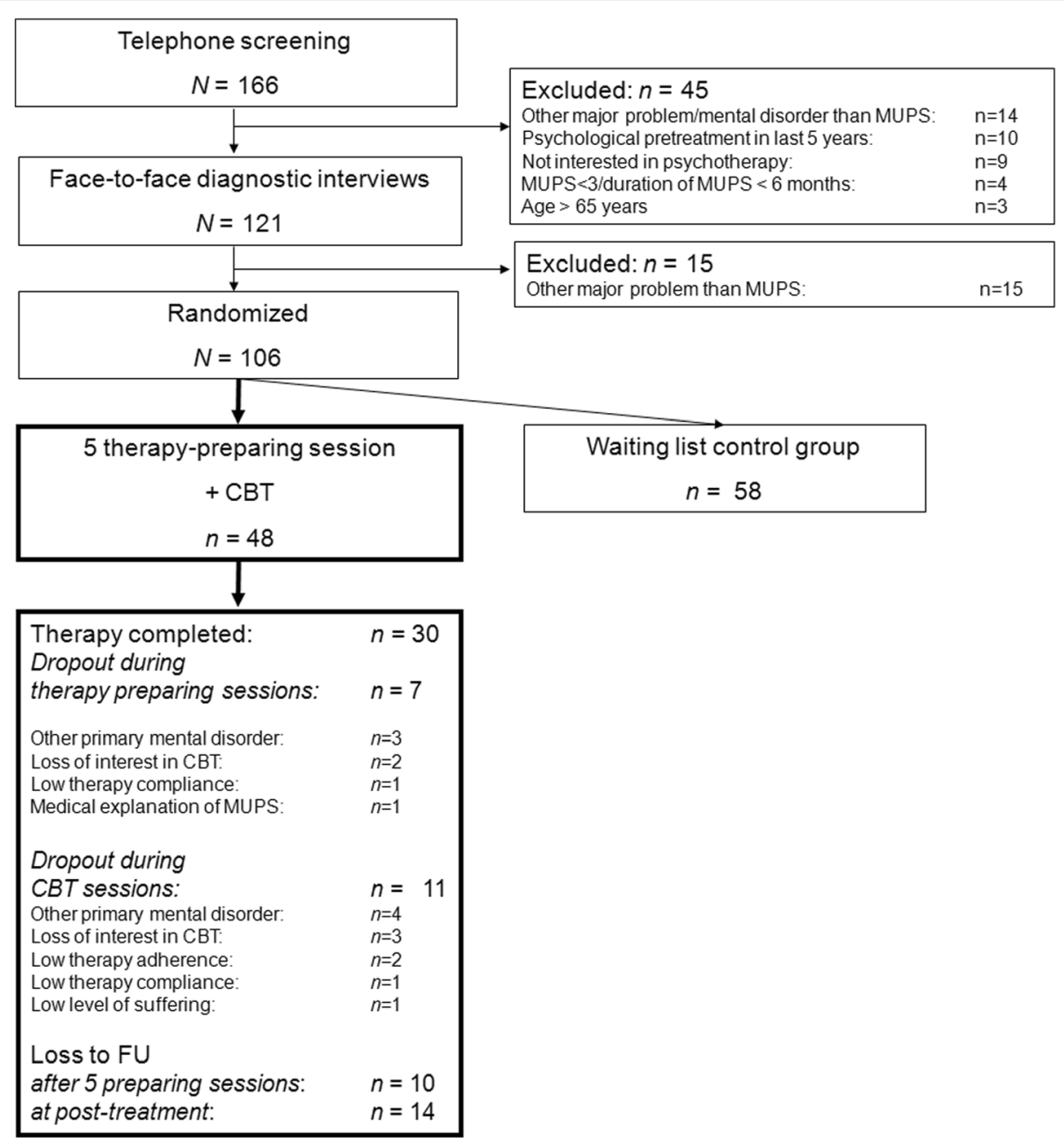

Fig. 1 Flow-chart of the RCT

of a somatization disorder, and $n=5(10.4 \%)$ of a pain disorder. Moreover $50.0 \%$ suffered from at least one comorbid disorder. On average individuals in the treatment group suffered 6.59 years $(S D=4.70$, range: 7 months - 25 years) from their somatic symptoms. Average Body Mass Index (BMI) was $23.11 \mathrm{~kg} / \mathrm{m}^{2}$ $\left(S D=4.40\right.$, range: $\left.16.54-35.66 \mathrm{~kg} / \mathrm{m}^{2}\right)$. As defined in the exclusion criteria of this trial, patients were not allowed to have psychological inpatient treatment during the last 5 years or psychological outpatient treatment addressing MUS during the last 2 years before starting the current study therapy. The general binary question if participants have ever attended a psychotherapy was answered with "no" by $64.4 \%(n=31)$. Pre-treatment scores of self-rating scales are depicted in Table 2.

Of the 48 participants $30(62.5 \%)$ completed the 5 therapy-preparing and 20 therapy sessions, 7 (14.6\%) quit therapy during the therapy-preparing sessions, and $11(22.9 \%)$ quit during the 20 therapy sessions. Reasons for therapy dropout are depicted in Fig. 1. Patients who completed therapy did not significantly differ from patients who dropped out of therapy in regard to demographic variables (age, sex, education, BMI), clinical variables (duration of somatic symptoms, comorbid mental disorders, and baseline scores of self-rating scales) $(p \geq .165)$. Moreover, 38 patients who completed questionnaires after the five therapy-preparing sessions did not significantly differ from patients who did not fill in questionnaires $(p \geq .085)$ in regard to the demographic and clinical variables, except for the BMI, $t(44)=-2.05, p=.046$. Patients who did not complete the questionnaires had a higher BMI $(M=25.55$, $S D=5.71)$ than patients who completed the self-rating scales $(M=22.43, S D=3.78)$. A similar result was found when 34 patients who completed questionnaires at posttreatment were compared with 14 patients who did not complete self-rating scales $(p \geq 256)$. There was only a difference in regard to educational level: group of 
Table 2 Mean values, standard deviations at pre-treament, after 5 therapy-preparing sessions, and after 20 therapy sessions and effect sizes

\begin{tabular}{|c|c|c|c|c|c|c|}
\hline \multirow[t]{2}{*}{ Outcome } & \multicolumn{3}{|l|}{$M(S D)$} & \multicolumn{3}{|l|}{$d(95 \% \mathrm{Cl})$} \\
\hline & Baseline & 5th session & 20th session & FU-1 vs. baseline & FU-2 vs. FU-1 & FU-2 vs. baseline \\
\hline SOMS-7 T & $0.62(0.31)$ & $0.63(0.38)$ & $0.55(0.36)$ & $-0.03[-0.43,0.37]$ & $0.22[-0.19,0.62]$ & $0.21[-0.19,0.61]$ \\
\hline BDI-II & $0.69(0.35)$ & $0.58(0.30)$ & $0.48(0.37)$ & $0.34[-0.07,0.74]$ & $0.30[-0.11,0.70]$ & $0.58[0.17,1.00]$ \\
\hline BSI-anxiety & $0.95(0.59)$ & $0.64(0.43)$ & $0.62(0.59)$ & $0.60[0.18,1.02]$ & $0.04[-0.36,0.44]$ & $0.56[0.14,0.97]$ \\
\hline IAS-illness anxiety & $1.65(0.86)$ & $1.33(0.81)$ & $1.06(0.69)$ & $0.38[-0.02,0.79]$ & $0.36[-0.05,0.77]$ & $0.76[0.33,1.18]$ \\
\hline IAS-illness behavior & $2.40(0.61)$ & $2.16(0.53)$ & $1.72(0.63)$ & $0.42[0.01,0.83]$ & $0.76[0.33,1.18]$ & $1.10[0.64,1.55]$ \\
\hline
\end{tabular}

SOMS-7 T Screening of Somatoform Disorders-7 T (answering scale: $0=$ not at all, $4=$ very strong), BDI-II Beck Depression Inventory-II (answering scale: 0-3), BSI Brief Symptom Inventory (answering scale: $0=$ not at all, $4=$ very strong), IAS Illness Attitude Scales (answering scale: $0=$ no, $4=$ most of the time)

patients who completed self-rating scales at FU-20 T were significantly higher educated than patients who did not complete questionnaires at FU-20 T, $\mathrm{Chi}^{2}(1$, $N=47)=5.43, p=.025$.

\section{Changes during 5 therapy-preparing sessions}

RmANOVAs revealed a significant change between pretreatment and FU-5P for the BDI, $F(1,47)=6.68$, $p=.014$, the anxiety subscale of BSI, $F(1,47)=9.86$, $p=.004$, for the illness anxiety subscale, $F(1,47)=13.59$, $p=.001$, and illness behavior subscale of IAS, $F(1,47)=8.30, p=.007$, but not for SOMS-7 T, $F(1,47)=0.05, p=.828$. Results indicated that depressive and anxiety symptoms, illness anxiety, as well as illness behavior significantly declined during the first 5 therapypreparing sessions with small to medium effect sizes (see Table 2), whereas somatic symptom severity did not change. Table 2 summarizes mean values and standard deviations for pre-treatment scores and FU-5P as well as effect sizes.

\section{Prediction of outcome at the end of therapy by pre-treatment values and changes during therapy- preparing sessions}

Hierarchical linear multiple regression analyses were conducted for the outcomes for which we found significant changes during the 5 therapy-preparing sessions: depression, anxiety, illness anxiety, and illness behavior. In all regression analyses the pre-treatment score was a significant predictor of the outcome at the termination of therapy, in the first and the second model of the analysis. The results indicated that high pre-treatment values were associated with high values at the end of treatment. For depression and illness anxiety the differential score between pre-treatment and FU-5P became an additional significant predictor in model 2 and explained additionally $12.1 \%, \mathrm{~F}(1,45)=8.01, p=.007$, and $10.0 \%, \mathrm{~F}(1,45)=7.46, p=.009$, respectively of the variance of the outcome at the end of therapy. Results indicated that a reduction of outcome between pretreatment and FU-5P was associated with lower values on the self-rating scales at FU-20 T. In contrast, for anxiety and illness behavior the differential score between pre-treatment and FU-5P did not become an additional significant predictor in model 2. The differential score explained additionally only $5.0 \%, F(1,45)=2.97$, $p=.092$, and $5.3 \%, F(1,45)=3.53, p=.067$, respectively of the variance of outcome at the end of therapy. Test statistics of the regression analyses are summarized in Table 3.

\section{Differences $\mathrm{RCl}+$ and $\mathrm{RCl}-$}

For the BDI only 2 (4.2\%) and for illness behavior subscale of IAS only 1 patient (2.1\%) reached a RCI. Consequently, differences between $\mathrm{RCI}+$ and RCI- were analyzed only for the BSI anxiety subscale and the IAS illness anxiety subscale since they were the only outcomes for which a sufficient number of participants reached a reliable change.

Regarding the BSI anxiety subscale an improvement of at least 0.94 was necessary to reach a reliable change. We observed this reliable change during the 5 therapypreparing sessions in $6(12.5 \%)$ patients. A mixed-effect $2 \times 3$ ANOVA resulted in a significant main effects of time, $F(2,92)=23.49, p<.001$, and group, $F(1,46)=10.21, p=.003$, and a significant time ${ }^{*}$ group interaction effect, $F(2,92)=13.38, p<.001$. Bonferronicorrected post-hoc comparisons were calculated in order to break up the significant interaction effect. They showed that RCI+ group had significantly higher anxiety values than the RCI- group at pre-treatment, $p<.001$, $g=2.53$ (95\% CI: 1.53, 3.52), whereas at FU-5P and FUT20 no significant between-group differences were found ( $p \geq .349)$. In the $\mathrm{RCI}+$ group a significant decline of anxiety symptoms between pre-treatment and FU-5P, $p<.001, d=2.71$ (95\% CI: $0.81,4.62)$ or FU-20 T, $p<.001, d=2.69$ (95\% CI: 0.79, 4.58) was found. The remaining inner-subject cell comparisons did not reveal further significant effects $(p \geq .069)$. Mean values, standard deviations, and effect sizes are summarized in Fig. 2.

Regarding the IAS illness anxiety subscale an improvement of 0.64 points indicated a reliable change. During 
Table 3 Test statistics of regression analyses of predictions of outcome at therapy termination by pre-treatment scores and changes during therapy-preparing sessions

\begin{tabular}{|c|c|c|c|c|c|}
\hline & $B$ & $\beta$ & $R^{2}$ & $F$ & $p$ \\
\hline \multicolumn{6}{|l|}{ Depression } \\
\hline Model $1^{\mathrm{a}}$ & & & .183 & $10.39^{* *}$ & .002 \\
\hline BDI-II (pre-treatment) & .37 & $.43^{* *}$ & & & .002 \\
\hline Model $2^{b}$ & & & .305 & $10.41^{* *}$ & .001 \\
\hline BDI-II (pre-treatment) & .57 & $.66^{* * *}$ & & & $<.001$ \\
\hline BDI-II ( $\triangle$ FU-5P - pre-treatment) & -.50 & $-.42^{* *}$ & & & .013 \\
\hline \multicolumn{6}{|l|}{ Anxiety } \\
\hline Model $1^{\mathrm{a}}$ & & & .161 & $8.87^{* *}$ & .002 \\
\hline BSI-anxiety (pre-treatment) & .34 & $.40^{* *}$ & & & .004 \\
\hline Model $2^{b}$ & & & .211 & $5.843^{* *}$ & .006 \\
\hline BSI-anxiety (pre-treatment) & .56 & $.67^{*}$ & & & .018 \\
\hline BSI-anxiety ( $\triangle \mathrm{FU}-5 \mathrm{P}$ - pre-treatment) & -.26 & -.33 & & & .235 \\
\hline \multicolumn{6}{|l|}{ Illness anxiety } \\
\hline Model $1^{a}$ & & & .291 & $18.99^{* *}$ & .002 \\
\hline IAS-illness anxiety (pre-treatment) & .44 & $.54^{* * *}$ & & & $<.001$ \\
\hline Model $2^{b}$ & & & .391 & $16.84^{* * *}$ & $<.001$ \\
\hline IAS-illness anxiety (pre-treatment) & .55 & $.68^{* * *}$ & & & $<.001$ \\
\hline IAS-illness anxiety ( $\triangle$ FU-5P - pre-treatment) & -.40 & $-.34^{*}$ & & & .016 \\
\hline \multicolumn{6}{|l|}{ Illness behavior } \\
\hline Model $1^{\mathrm{a}}$ & & & .271 & $17.34^{* *}$ & .002 \\
\hline IAS-illness behavior (pre-treatment) & .51 & $.52^{* * *}$ & & & $<.001$ \\
\hline Model $2^{b}$ & & & .323 & $10.71^{* * *}$ & $<.001$ \\
\hline IAS-illness behavior (pre-treatment) & .70 & $.71^{* * *}$ & & & $<.001$ \\
\hline IAS-illness behavior ( $\triangle \mathrm{FU}-5 \mathrm{P}$ - pre-treatment) & -.32 & -.29 & & & .135 \\
\hline
\end{tabular}

SOMS-7 T Screening of Somatoform Disorders-7 T, BDI Beck Depression Inventory-II, BSI Brief Symptom Inventory, IAS IIIness Attitude Scales

${ }^{\mathrm{a}} d f_{\mathrm{n}}=1, d f_{\mathrm{d}}=46{ }^{\mathrm{b}} d f_{\mathrm{n}}=2, d f_{\mathrm{d}}=45$

${ }^{*} p<.05,{ }^{* *} p<.01,{ }^{* * *} p<.001$

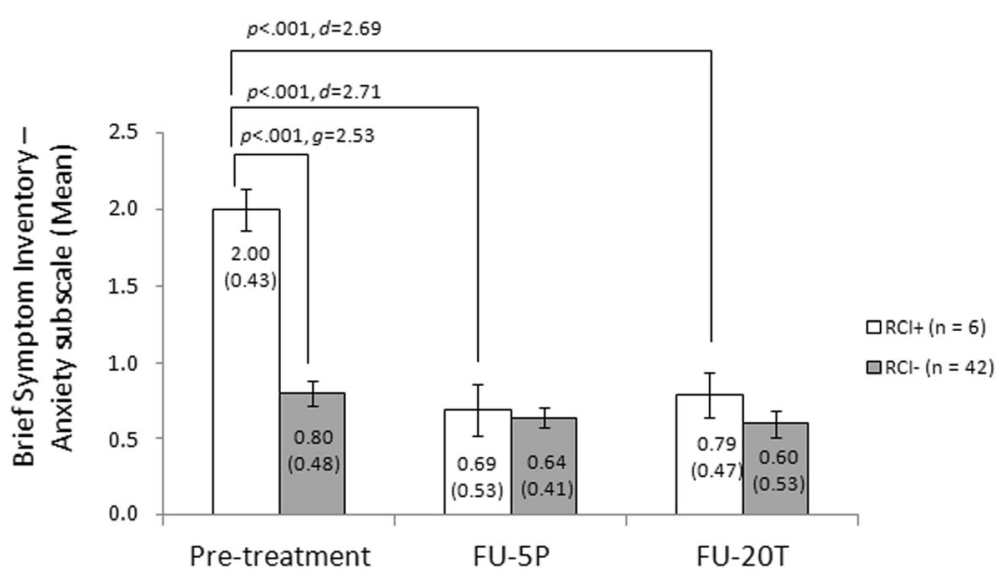

Assessment

Fig. 2 Mean values and standard deviations at pre-treatment, follow-up after 5 therapy-preparing sessions (FU-5P) and 20 therapy sessions (FU-20 T), between-group and inner-subject effect sizes and test statistics for the anxiety subscale of the Brief Symptom Inventory (separately for patients who reached a clinically significant decline of anxiety symptoms after 5 therapy-preparing sessions $[\mathrm{RCl}+]$ versus patients who did not $[\mathrm{RCI}-])$ 
the 5 therapy-preparing sessions 10 patients (20.8\%) reached the RCI. A mixed-effect $2 \times 3$ ANOVA resulted in a significant main effect of time, $F(2,92)=16.31$, $p<.001$. However the main effect of group, $F(1,46)=1.40, p=.244$, did not reach significance, and also the time"group interaction effect slightly failed to reach significance, $F(2,92)=3.01, p<.054$. Bonferronicorrected post-hoc comparisons showed only one significant between-group difference, at FU-5P: $\mathrm{RCI}+$ had significantly lower illness anxiety scores than RCI- group, $p=.041, g=0.75$ (95\% CI: 0.04, 1.46). Moreover posthoc comparisons showed that in the RCI+ group a significant decline of illness anxiety took place between pre-treatment and FU-5P, $p<.001, d=1.22$ (95\% CI: $0.20,2.25)$, and between pre-treatment and FU-T20, $p=.007, d=1.60$ (95\% CI: 0.48, 2.72). In contrast in the RCI- group the significant decrease of illness anxiety was found between FU-5P and FU-20 T, $p=.014$, $d=0.41$ (95\% CI: $-0.05,0.87)$ and between pretreatment and FU-20 T, $p<.001, d=0.62$ (95\% CI: 0.15 , 1.09). Mean values, standard deviations and effect sizes are summarized in Fig. 3.

\section{Relationships between changes during the 5 therapy- preparing sessions and demographic or clinical variables at baseline}

We calculated RGSs based on the difference between pre-treatment and FU-5P for variables which demonstrated a significant change the five therapy-preparing sessions: BDI, BSI anxiety subscale, IAS illness anxiety and illness behavior subscale. The Pearson productmoment and Spearman rank correlation coefficients are summarized in Table 4. None of the correlation coefficients reached significance.

\section{Discussion}

The central aims of the current study were first to examine early symptom changes in patients with medically unexplained symptoms (MUS) during five therapypreparing sessions. Secondly, we wanted to find out if these early changes are predictors of therapy outcome at therapy termination, after controlling for pre-treatment scores. The third aim was to investigate differences between patients who reached a reliable change after the therapy-preparing phase with patients who did not. Finally we examined if early changes in CBT for somatoform disorders are associated with demographic and clinical variables. Most important findings are summarized and discussed in the following.

The first important result is that although the severity of patients' somatic symptoms did not change during the therapy-preparing phase, patients experienced a significant decline of depressive symptoms, anxiety, illness anxiety and illness behavior. In comparison to other studies of early response in psychotherapy the rates of responders was however rather small. The highest rate of patients who reached a reliable change in the first 5 sessions was found for illness anxiety (10 of 48 patients, $20.8 \%$ ) and for anxiety (6 of 48 patients, $12.5 \%$ ). Innersubject effect sizes of these early changes were small to moderate across outcomes. Our findings are similar to a previous study which examined early response in patients suffering from irritable bowel syndrome (IBS) [27]. Authors of this previous study found a similar rate of

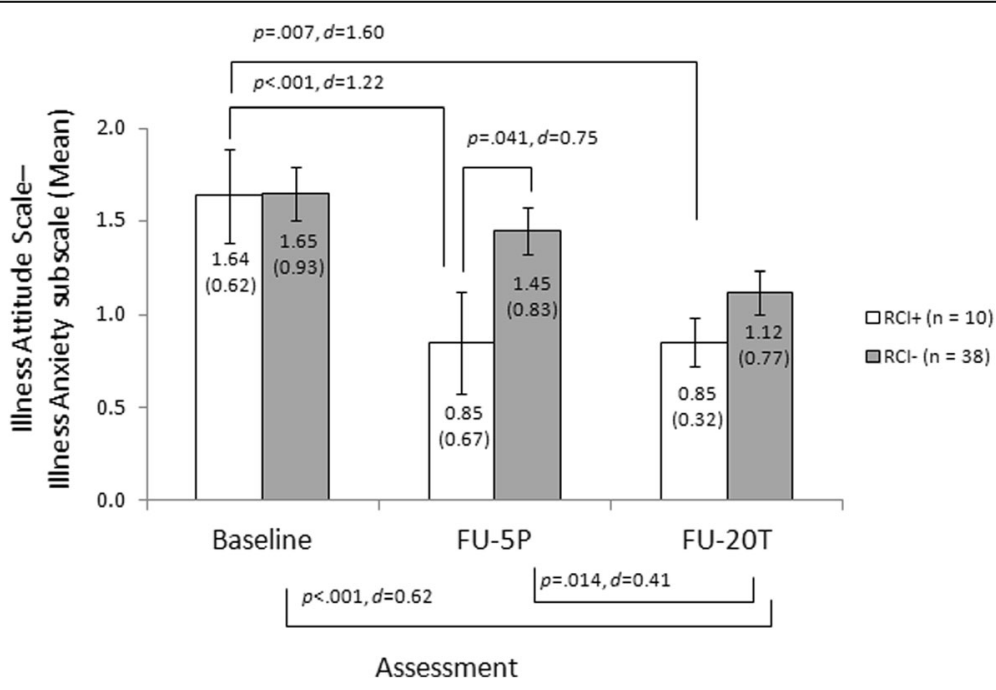

Fig. 3 Mean values and standard deviations at pre-treatment, follow-up after 5 therapy-preparing sessions (FU-5P) and 20 therapy sessions (FU-20 T), between-group and inner-subject effect sizes and test statistics for the illness anxiety subscale of the Illness Attitude Scales (separately for patients who reached a clinically significant decline of illness anxiety symptoms after 5 therapy-preparing sessions [RCl+] versus patients who did not $[\mathrm{RCl}-])$ 
Table 4 Pearson product-moment and Spearman rank correlation coefficients between RGS scores and demographic and clinical variables, and baseline scores of self-rating scales

\begin{tabular}{|c|c|c|c|c|}
\hline & \multicolumn{4}{|l|}{$r(p)$} \\
\hline & $\begin{array}{l}\text { RGS } \\
\text { BDI }\end{array}$ & $\begin{array}{l}\text { RGS } \\
\text { BSI-A }\end{array}$ & $\begin{array}{l}\text { RGS } \\
\text { IAS-IA }\end{array}$ & $\begin{array}{l}\text { RGS } \\
\text { IAS- IB }\end{array}$ \\
\hline$\overline{S e x^{a}}$ & $.01(.957)$ & $-.05(.763)$ & $-.11(.453)$ & $.01(.946)$ \\
\hline Age & $.18(.240)$ & $-.06(.717)$ & $.03(.825)$ & $.08(.608)$ \\
\hline Education $^{a}$ & $-.11(.495)$ & $.02(.989)$ & $.04(.777)$ & $.06(.719)$ \\
\hline BMI & $-.03(.832)$ & $.03(.861)$ & $.17(.282)$ & $-.20(.309)$ \\
\hline Duration of somatic symptoms & $-.22(.178)$ & $-.23(.162)$ & $-.23(.129)$ & $-.09(.563)$ \\
\hline Comorbidity $^{a}$ & $-.16(.279)$ & $-.16(.297)$ & $.23(.129)$ & $.10(.541)$ \\
\hline SOMS-7 T (Baseline) & $-.12(.441)$ & $-.04(.792)$ & $.09(.537)$ & $-.14(.383)$ \\
\hline BDI (Baseline) & - & $-.01(.950)$ & $.05(.767)$ & $-.01(.943)$ \\
\hline BSI-A (Baseline) & $.11(.473)$ & - & $.09(.545)$ & $-.00(.989)$ \\
\hline IAS-IA (Baseline) & $.14(.383)$ & $-.06(.685)$ & - & $-.03(.859)$ \\
\hline IAS-IB (Baseline) & $-.03(.862)$ & $.02(.895)$ & $.08(.597)$ & - \\
\hline
\end{tabular}

SOMS-7 T Screening of Somatoform Disorders-7 T, BDI Beck Depression Inventory-II, BSI-A Brief Symptom Inventory - Anxiety subscale, IAS-IA/-IB IIIness Attitude Scales - illness anxiety subscale/illness behavior subscale, BMI Body Mass Index

${ }^{a}$ Spearman rank correlation coefficients

early response $(30 \%$ of the participants fulfilled criteria of early response after the 4th therapy week) [27]. In contrast to our study, Lackner, Gudleski, Krasner, Powell, and Katz [27] found however an early response effect also in regard to somatic symptoms. However it has to be considered that in the mentioned study [27] patients received the therapeutic interventions immediately the first therapy session. There were no therapypreparing sessions like in our study.

In studies based on patients with other mental disorders early response rates are higher. For example in the study by Fennell and Teasdale [4] 47.1\% of a depressive sample showed a decrease of depressive symptoms of $50 \%$ or more. In the study by Renaud et al. [5] 31.0\% had an improvement of at least 50\% during the first two therapy sessions. However it has to be considered that meta-analyses demonstrated CBT to be not as effective for MUS [19] as for depression [49]. Moreover our results are difficult to compare with other previous studies since they do not only differ in regard to participants' diagnoses but also in regard to the number of sessions being considered for the definition of early response (e.g. 2 sessions in the studies by Fennell and Teasdale [4] and Renaud [5] vs. 5 sessions in our study) and the definition of early response (e.g. reaching $\mathrm{RCI}$ in our sample vs. a symptoms' decrease of $50 \%$ or more in the study by Fennell and Teasdale [4]). Erekson, Lambert, and Horner [50] have shown that the different methods of defining dramatic change produce large discrepancies in rates of individuals meeting criteria. These ranged from $42 \%$ to $5 \%$ based on a single measure and a single sample of outpatients with mixed diagnoses. Furthermore, our study differed from previous studies in regard to contents of the early sessions. Whereas in our study the first five sessions had a therapy-preparing character and did not include any CBT interventions, in other studies therapeutic interventions already took place during the early treatment phase.

An important question is how this change in affectivecognitive and behavioral outcomes in a sample of patients with long-standing and distressing MUS can be explained. It could be speculated if this result indicates a kind of "placebo effect". The concept of "placebo effect" comes from pharmacological research and is by definition "the effect that occurs following administration of a placebo, that is, an inert treatment" [51]. However this definition is very restrictive when placebo-like effects in which no placebo is given are considered [52] and can be applied to psychotherapy research only to a limited extend. These effects are rather attributable to the impact of the context factors surrounding the treatment [51] such as different kinds of patients' expectations [53]. Accordingly, Lambert [54] assumes that early response patterns in psychotherapy are rather evidence for common factors than a "placebo effect" in its original medical meaning. Common factors are those dimensions of the treatment setting (e.g., therapist, client) which are not specific to a particular therapeutic technique. As already mentioned in pharmacotherapy early response is rather considered as a "premature" change in symptomatology associated with a final negative therapy outcome and risk of relapse [2, 3]. In contrast, in psychotherapy previous research however has already demonstrated that across different forms of therapy early response seems to be associated with a positive therapy outcome at termination and follow-up [6, 7]. 
Ilardi and Craighead [29] examined several studies of CBT for depression in regard to early responses. They explained the phenomenon by the impact of common factors such as the formation of a collaborative therapeutic alliance, the scheduling of frequent and regular appointments at a designated treatment setting, the formal presentation of a treatment rationale, and the introduction of an explicit procedure touted as integral to the recovery process - this means common factors which cannot be attributed to specific therapy techniques. These assumptions would fit very well with our findings. After many years of repeated, mainly ineffective medical diagnostic procedures and treatments associated with the experience of disappointment and frustration as well as the repeated experience of less validating communication with the clinician, it can be assumed that the first psychotherapy sessions are a completely new experience to the prototypic somatoform patient. In CBT for somatoform patients these first therapy-preparing sessions are usually filled with actively listening to the patient and validating the suffering associated with somatic complaints [55] in order to establish a trustful patient-therapist relationship. The patient shall feel understood and taken seriously. Moreover patients experience a kind of reassuring effect during these first sessions which could be based on simply knowing that they will see a professional that is there to help them every week. This could contribute to reducing their anxiety. However at it has been demonstrated in previous research this effect of reassurance would likely not last in the absence of an actual therapy especially in regard to illness anxiety. This could be explained by the result of another study which demonstrated that patients with MUS (in contrast to patients with depression and healthy subjects) remembered a higher likelihood for medical explanations for their symptoms and reported more concerns about their health state [56].

The aim of these first therapy-preparing sessions is to provide the patient with the expectation that psychotherapy could be a helpful intervention. Moreover patients' biomedical causal symptom attributions are slowly extended to psychosocial factors. It would make sense that this new interpersonal experience can have a strong impact especially on cognitive-affective variables such as anxiety and depressive symptoms. Although we cannot make any statements about which processes exactly cause changes in these first five sessions, this would be an important question for future research. In CBT for depression Fennell and Teasdale [4] have already found out that early responders engage in therapy in a different manner than later responders. For example early responders appeared to proceed from one problem to the next in therapy in a sequential pattern, whereas delayed responders continued to revisit the same therapy topic across sessions.
A second important finding is that early improvements of depressive symptoms and illness anxiety during the 5 therapy-preparing sessions are predictors of a better outcome at the end of therapy, after controlling for pretreatment scores. This result is in accordance with the predictive value of early response patterns demonstrated for IBS [27] and other mental disorders [4, 6]. Haas et al. [6] for example found the same pattern of results in a large sample of psychotherapy outpatients with mixed diagnoses. Fennell and Teasdale [4] demonstrated similar predictions of the outcome of the CBT by early response in a sample of depressive patients.

The third important finding of our study refers to the maintenance of symptom improvement after 5 therapypreparing sessions until the termination of therapy. In regard to anxiety and illness anxiety we identified a sufficient number of patients who reached a reliable change after the therapy-preparing phase. Contrasting them with patients who did not reach the RCI resulted in two important findings. First, between-group differences were dependent on the kind of outcome. Regarding anxiety, especially patients with high pre-treatment scores showed a strong decrease of anxiety symptoms and maintained this until the termination of therapy. They differed from patients who did not reach the RCI, in regard to pre-treatment scores but not in regard to scores after the therapy-preparing sessions and at the end of therapy. In the contrary for illness anxiety there was another pattern. Patients who reached RCI during the therapy-preparing sessions maintained this improvement until the end of therapy. Patients who did not reach RCI during the 5 preparing sessions improved significantly during the 20 therapy sessions. Both groups only differed significantly after the five therapy-preparing sessions, but not at pre-treatment or at therapy termination. Secondly, for both outcomes early response could be maintained until the end of therapy. This has also been demonstrated for IBS [27] and depressive [4, 5] as well as samples of patients with mixed diagnoses of mental disorders [6]. In contrast to the mentioned studies we could however not demonstrate in our somatoform sample that patients with an early response have a better outcome at therapy termination. However our results are just preliminary and are based on very low sample sizes. Therefore they have to be interpreted cautiously.

A final important finding of our study is that we could not identify any demographic or clinical variables being associated with early changes in therapy. These zeroorder correlations have also been demonstrated in a sample of depressive patients receiving CBT in a previous trial [4]. However we could show that higher pretreatment values on a self-rating scale were significantly associated with bigger changes on this scale after the five therapy-preparing sessions. This finding is similar to 
results of a study examining early response in IBS [27]. In our study the highest correlation was found in regard to anxiety: high pretreatment anxiety scores were associated with a stronger decline of anxiety in the therapypreparing phase. This result can be possibly explained since these five early sessions are filled with establishing a therapy alliance. The bonding experience can probably have an anxiolytic effect on patients, for example by providing them with reassurance or emotional support and empathy [57]. Of course, it can be assumed that early improvement of symptoms can also have a reverse effect and can improve therapy alliance [58]. Besides the study by Fennell and Teasdale [4] mentioned above, there is only one further study where correlates of early response were investigated. Lutz et al. [8] identified depressive and interpersonal problems, agoraphobic and somatization symptoms, and problems at school or in the job as predictors.

To our knowledge the current study is the first trial which provides important findings in regard to the role of early response in the psychotherapy of somatoform disorders characterized by multiple MUS. For the interpretation of our findings the following limitations of our study however should be considered. First of all, sample size was small and missing rates were high. Although we analyzed differences regarding baseline variables between patients who dropped out and those who completed therapy, we failed to assess other important variables (e.g. pre-treatment therapy motivation) which could be associated with post-treatment results and which could account for dropout rates skewing our findings. Another limitation was that we assessed therapy progress only three times (pre-treatment, FU-5P, and FU-20 T). Process measures which are filled in by patients would be more appropriate in order to examine early response patterns. Moreover due to the failures in the implementation of the study we were not able to consider the control group in our analyses and to compare the control with the CBT data. However we can at least conclude from previous intervention studies that the kind of CBT we provided to our patients has been proven to be effective in comparison to a wait-list control groups $[19,20]$.

\section{Conclusions}

It is important to emphasize that finally it should not be concluded from our data that "just listening" and "validating symptom-related distress" is sufficient to treat patients with chronic MUS. It is not realistic to expect that the kinds of problems of patients who suffer over many years from distressing and impairing somatic symptoms can be solved within five sessions and that these effects are long-lasting. Our data should be rather interpreted as evidence that changes during these early sessions have a strong predictive value for long-term outcomes in somatoform patients. Although we do not know exactly which mechanisms cause early response patterns it makes sense to assume that new experiences in the therapeutic relationship can already have healing effects in patients with MUS, especially under consideration of patients' typical previous interpersonal experiences in patient-clinician relationships. This assumption would especially fit specific early responses in regard to affective-emotional variables in contrast to somatic symptom severity. Wampold [59] postulates in his contextual model of psychotherapy that an initial bond between patient and therapist is an important basis for making therapy work and for initiating important therapeutic processes - such as the stepwise extension of MUS-patients' biomedical causal symptom attributions to psychosocial factors.

Future studies are needed to replicate our findings with larger sample sizes and including active control conditions. These studies should also apply assessments not only after single but after every session in order to monitor the early response pattern and therapy process session by session. Moreover an important conclusion is that future research should not only focus on somatic symptom severity, as it has happened in almost all previous intervention studies on somatoform patients $[19,20]$, but also include symptom-related outcomes. It has to be emphasized that reducing symptom severity is in fact not the primary aim of CBT for somatoform disorders but rather improving symptom coping. In general it seems that research on early response patterns in psychotherapy should not only focus on primary outcomes [e.g., 4, 5, 7], since these patterns can possibly vary dependent on which type of outcome is considered. We implemented all mentioned issues which are important for future research on therapy processes in CBT for MUS in a further large multicenter RCT in which we compare a conventional CBT with a CBT enriched with emotion regulation strategies for somatoform patients. Further details of this study can be taken from the study protocol [60]. We hope that we can move forward with gaining more insight into early response patterns and its mechanisms in somatoform patients.

\footnotetext{
Abbreviations

(rm)ANOVA: (repeated measure) analysis of variance; BDI-II: Beck Depression Inventory-II; BMI: Body Mass Index; BSI: Brief Symptom Inventory; CBT: Cognitive-behavior therapy; DSM-IV/-5: Diagnostic and Statistical Manual for Mental Disorders, 4th/5th revision; FU-5P/-20 T: Follow-up assessment after 5 therapy-preparing sessions/20 therapy sessions; IBS: Irritable bowel syndrome; ICD-10: International Classification of Diseases, 10th revision; MDD: Major depressive disorder; MUS: Medically unexplained symptoms; RCl: Reliable change index; $\mathrm{RCl}+$ /-: Subgroup which fulfilled/ did not fulfill the reliable change index; RCT: Randomized controlled trial; RGS: Residual gain score; SOMS-7 T: Screening for Somatoform Disorders-7 T
} 


\section{Acknowledgements}

Special thanks to the Ann-Christin Krämer-Heil, Christine Serwe, Petra Thomas, Carina Grama, and Fabian Jasper who supported us with diagnostic interviews and recruiting patients.

\section{Funding}

There was no funding for the study.

\section{Availability of data and materials}

The datasets during and/or analyzed during the current study available from the corresponding author on reasonable request.

\section{Authors' contributions}

MK analyzed data and wrote the first draft of the paper. All authors interpreted data. $\mathrm{ML}$ and $\mathrm{WH}$ supervised the preparation of the manuscript. All authors read and approved the final manuscript. ML proofread the final manuscript.

\section{Competing interests}

The authors declare that they have no competing interests.

\section{Consent for publication}

Not applicable.

\section{Ethics approval and consent to participate}

The study was approved by the Ethics Committee of the German Psychological Association (DGPs; reference number: WH28092006DGPS; approval date: 29 October 2006). Each patient had to sign informed consent during the diagnostic interview before random assignment.

\section{Publisher's Note}

Springer Nature remains neutral with regard to jurisdictional claims in published maps and institutional affiliations.

\section{Author details}

'Division of Clinical Psychology and Psychotherapy, Philipps-University, Gutenbergstr. 18, D-35037 Marburg, Germany. ${ }^{2}$ Psychological Medicine, School of Medicine, Faculty of Medical and Health Sciences, University of Auckland, Hospital Bldg. 599, 2 Park Rd, Grafton Auckland, Auckland 1023, New Zealand. ${ }^{3}$ Department of Psychology, Brigham Young University, Provo, USA. ${ }^{4}$ Department of Clinical Psychology and Psychotherapy, Johannes Gutenberg-University, Mainz, Germany.

Received: 23 October 2016 Accepted: 5 May 2017

Published online: 25 May 2017

\section{References}

1. Nierenberg AA, Quitkin FM, Kremer C, Keller MB, Thase ME. Placebocontrolled continuation treatment with mirtazapine: acute pattern of response predicts relapse. Neuropsychopharmacology. 2004;29:1012-8.

2. Quitkin FM, Rabkin JD, Markowitz JM, Stewart JW, Mcgrath PJ, Harrison W. Use of pattern-analysis to identify true drug response - a replication. Arch Gen Psych. 1987;44:259-64.

3. Stewart JW, Tricamo E, McGrath PJ, Quitkin FM. Prophylactic efficacy of phenelzine and imipramine in chronic atypical depression: likelihood of recurrence on discontinuation after 6 months' remission. Am J Psychiat. 1997;154:31-6

4. Fennell MJV, Teasdale JD. Cognitive therapy for depression - individua differences and the process of change. Cognitive Ther Res. 1987;11:253-71.

5. Renaud J, Brent DA, Baugher M, Birmaher B, Kolko DJ, Bridge J. Rapid response to psychosocial treatment for adolescent depression: a two-year follow-up. J Am Acad Child Psy. 1998;37:1184-90.

6. Haas E, Hill RD, Lambert MJ, Morrell B. Do early responders to psychotherapy maintain treatment gains? J Clin Psychol. 2002;58:1157-72.

7. Lutz W, Stulz N, Köck K. Patterns of early change and their relationship to outcome and follow-up among patients with major depressive disorders. J Affect Disorders. 2009:118:60-8.

8. Lutz W, Stulz N, Smart DW, Lambert MJ. Patterns of early change in outpatient therapy [Die Identifikation früher Veränderungsmuster in der ambulanten Psychotherapie]. Z KI Psych Psychoth. 2007;36:93-104.
9. Sharpe M, Mayou R, Bass C: Concepts, theories and terminology. In Treatment of functional somatic symptoms. Edited by Mayou R, Bass C, Sharpe M. Oxford, UK: Oxford University Press; 1995: 3-16.

10. American Psychiatric Association: Diagnostic and statistical manual for mental disorders. Fourth edition, text revision. Washington, DC: American Psychiatric Press; 2000

11. American Psychiatric Association: Diagnostic and statistical manual of mental disorders. Fifth edition. DSM-5. Washington, DC: APA; 2013.

12. Jacobi F, Wittchen HU, Holting C, Hofler M, Pfister H, Muller N, Lieb R. Prevalence, co-morbidity and correlates of mental disorders in the general population: results from the German health interview and examination survey (GHS). Psychol Med. 2004;34:597-611.

13. Steinbrecher $N$, Körber $S$, Frieser D, Hiller W. The prevalence of medically unexplained symptoms in primary care. Psychosomatics. 2011;52:263-71.

14. Nimnuan C, Hotopf M, Wessely S. Medically unexplained symptoms: an epidemiological study in seven specialities. J Psychosom Res. 2001:51:361-7.

15. Rief W, Rojas G. Stability of somatoform symptoms - implications for classification. Psychosom Med. 2007:69:864-9.

16. van Eck van der Sluijs JV, Ten Have M, Rijnders C, van Marwijk H, de Graaf R, van der Feltz-Cornelis C: Medically unexplained and explained physical symptoms in the general population: Association with prevalent and incident mental disorders. PLoS One 2015, 10.

17. de Waal MW, Arnold IA, Eekhof JA, van Hemert AM. Somatoform disorders in general practice: prevalence, functional impairment and comorbidity with anxiety and depressive disorders. Brit J Psychiat. 2004;184:470-6.

18. Barsky AJ, Orav EJ, Bates DW. Somatization increases medical utilization and costs independent of psychiatric and medical comorbidity. Arch Gen Psych. 2005;62:903-10.

19. Van Dessel N, Den Boeft M, Van der Wouden JC, Kleinstäuber M, Leone SS, Terluin B, Numans ME, Van der Horst HE, Van Marwijk H: Nonpharmacological interventions for somatoform disorders and medically unexplained physical symptoms (MUPS) in adults. Cochrane Database of Systematic Reviews 2014, Issue 11. Art. No.: CD011142. http://dx.doi.org/ 10.1002/14651858.CD011142.pub2.

20. Kleinstäuber M, Witthöft M, Hiller W. Efficacy of short-term psychotherapy for multiple medically unexplained physical symptoms: a meta-analysis. Clin Psychol Rev. 2011:31:146-60.

21. Martin A, Korn HJ, Cebulla M, Saly M, Fichter MM, Hiller W. Causal attributions about bodily sensations in somatoform disorders. Z Psychiatr Psych Ps. 2007;55:31-41

22. Murray AM, Toussaint A, Althaus A, Löwe B. The challenge of diagnosing non-specific, functional, and somatoform disorders: a systematic review of barriers to diagnosis in primary care. J Psychosom Res. 2016;80:1-10.

23. Wileman L, May C, Chew-Graham CA. Medically unexplained symptoms and the problem of power in the primary care consultation: a qualitative study. Fam Pract. 2002;19:178-82.

24. Ring A, Dowrick C, Humphris G, Salmon P. Do patients with unexplained physical symptoms pressurise general practitioners for somatic treatment? A qualitative study. Brit Med J. 2004;328:1057-60.

25. Salmon P, Humphris GM, Ring A, Davies JC, Dowrick CF. Primary care consultations about medically unexplained symptoms: patient presentations and doctor responses that influence the probability of somatic intervention. Psychosom Med. 2007;69:571-7.

26. McGorm K, Burton C, Weller D, Murray G, Sharpe M. Patients repeatedly referred to secondary care with symptoms unexplained by organic disease: prevalence, characteristics and referral pattern. Fam Pract. 2010;27:479-86.

27. Lackner JM, Gudleski GD, Krasner SS, Powell C, Katz LA. Rapid response to cognitive behavior therapy for irritable bowel syndrome. Clin Gastroenterol Hepatol. 2008:8:426-32.

28. Francis $\mathrm{CY}$, Morris J, Whorwell PJ. The irritable bowel severity scoring system: a simple method of monitoring irritable bowel syndrome and its progress. Aliment Pharmacol Ther. 1997;11:395-402.

29. Ilardi SS, Craighead WE. The role of non-specific factors in cognitivebehavior for depression. Clin Psychol-Sci Pr. 1994;1:138-56.

30. Ilardi SS, Craighead WE. Rapid early response, cognitive modification, and nonspecific factors in cognitive behavior therapy for depression: a reply to tang and DeRubeis. Clin Psychol-Sci Pr. 1999;6:295-9.

31. Janca A, Hiller W. ICD-10 checklists - a tool for clinicians' use of the ICD-10 classification of mental and behavioral disorders. Compr Psychiatry. 1996;37:180-7. 
32. Mewes R, Rief W, Stenzel N, Glaesmer H, Martin A, Brahler E. What is "normal" disability? An investigation of disability in the general population. Pain. 2009;142:36-41.

33. Faul F, Erdfelder E, Lang AG, Buchner A. G* power 3: a flexible statistical power analysis program for the social, behavioral, and biomedical sciences. Behav Res Methods. 2007:39:175-91.

34. First MB, Gibbon M, Spitzer RL, Williams JBW, Smith Benjamin L. User's guide for the structured clinical interview for DSM-IV Axis II personality disorders (SCID-II). Washington DC: American Psychiatric Press; 1997.

35. Kleinstäuber M, Thomas P, Witthöft M, Hiller W. Cognitive-behavioral therapy for medically unexplained physical symptoms and somatoform disorders [Kognitive Verhaltenstherapie bei medizinisch unerklärten Körperbeschwerden und somatoformen Störungen]. Berlin, Germany: Springer; 2012.

36. Rief W, Hiller W. A new approach to the assessment of the treatment effects of somatoform disorders. Psychosomatics. 2003;44:492-8.

37. World Health Organization: International statistical classification of diseases. 10th revision (ICD-10). Genf, Switzerland: WHO; 1996.

38. Beck AT, Steer RA, Brown GK. Beck depression Inventory II (BDI-II). Manual. San Antonio, TX: Psychological Corporation; 1996.

39. Derogatis LR. The Brief symptom Inventory (BSI): administration, scoring, and procedures manual II. Baltimore, MD: Clinical Psychometric Research; 1992.

40. Boulet J, Boss MW. Reliability and validity of the Brief symptom Inventory. Psychol Assessment. 1991:3:433-7.

41. Kellner R. Abridged manual of the illness Attitude scales. Albuquerque, NM: University of New Mexico, Department of Psychiatry, School of Medicine, Mimeographed; 1987.

42. Speckens AEM, Spinhoven P, Sloekers PPA. Bolk JH, vanHemert AM: a validation study of the whitely index, the illness Attitude scales, and the somatosensory amplification scale in general medical and general practice patients. J Psychosom Res. 1996;40:95-104.

43. Hiller W, Rief W, Fichter MM. Dimensional and categorical approaches to hypochondriasis. Psychol Med. 2002;32:707-18.

44. Jacobson NS, Truax P. Clinical-significance - a statistical approach to defining meaningful change in psychotherapy-research. J Consult Clin Psych. 1991;59:12-9.

45. Schafer JL. Analysis of incomplete multivariate data. New York: Chapman \& Hall; 2000.

46. Cohen J. A power primer. Psychol Bull. 1992;112:155-9.

47. Steketee $\mathrm{G}$, Chambless DL. Methodological issues in prediction of treatment outcome. Clin Psychol Rev. 1992;12:387-400.

48. United Nations Educational Scientific and Cultural Organization Institute for Statistics. International standard classification of education (ISCED 97). Paris (France): United Nations Educational Scientific and Cultural Organization; 2006.

49. Cuijpers $P$, van Straten A, Bohlmeijer E, Hollon SD, Andersson G. The effects of psychotherapy for adult depression are overestimated: a meta-analysis of study quality and effect size. Psychol Med. 2010;40:211-23.

50. Erekson D, Lambert MJ, Horner J. Different lens or different picture? Psychother Res: Comparing methods of defining dramatic change in psychotherapy; in press.

51. Benedetti F. Mechanisms of placebo and placebo-related effects across diseases and treatments. Eur Neuropsychopharmacol. 2010;20:S60-1.

52. Moerman DE. Meaning, medicine and the placebo effect. Cambridge, MA: Cambridge Univ. Press; 2002.

53. Colloca L, Miller FG. Role of expectations in health. Curr OpinPsychiatr. 2011;24:149-55.

54. Lambert MJ. Early response in psychotherapy: further evidence for the importance of common factors rather than "placebo effects". J Clin Psychol. 2005;61:855-69.

55. Guidance for health professionals on medically unexplained symptoms (MUS) [http://www.rcpsych.ac.uk/pdf/CHECKED\%20MUS\%20Guidance_A4_4pp_6.pdf].

56. Rief W, Heitmüller $A M$, Reisberg $K$, Rüddel $H$. Why reassurance fails in patients with unexplained symptoms - an experimental investigation of remembered probabilities. PLoS Med. 2006:3:1266-72.

57. Linton SJ, McCracken LM, Vlaeyen JWS. Reassurance: help or hinder in the treatment of pain. Pain. 2008;134:5-8.

58. Turner H, Bryant-Waugh $\mathrm{R}$, Marshall E. The impact of early symptom change and therapeutic alliance on treatment outcome in cognitive-behavioural therapy for eating disorders. Behav Res Ther. 2015;73:165-9.
59. Wampold BE. How important are the common factors in psychotherapy? An update. World Psychiatry. 2015;14:270-7.

60. Kleinstäuber M, Gottschalk J, Berking M, Rau J, Rief W. Enriching cognitive behavior therapy with emotion regulation training for patients with multiple medically unexplained symptoms (ENCERT): design and implementation of a multicenter, randomized, active-controlled trial. Contemp Clin Trials. 2016;47:54-63.

\section{Submit your next manuscript to BioMed Central and we will help you at every step:}

- We accept pre-submission inquiries

- Our selector tool helps you to find the most relevant journal

- We provide round the clock customer support

- Convenient online submission

- Thorough peer review

- Inclusion in PubMed and all major indexing services

- Maximum visibility for your research

Submit your manuscript at www.biomedcentral.com/submit 